\title{
Validation of spectral sky radiance derived from all-sky camera images - a case study
}

\author{
K. Tohsing, M. Schrempf, S. Riechelmann, and G. Seckmeyer \\ Institut für Meteorologie und Klimatologie, Leibniz Universität Hannover, Herrenhäuser Straße 2, Hanover 30419, Germany \\ Correspondence to: K. Tohsing (korntip@ su.ac.th)
}

Received: 30 September 2013 - Published in Atmos. Meas. Tech. Discuss.: 10 January 2014

Revised: 5 May 2014 - Accepted: 23 May 2014 - Published: 16 July 2014

\begin{abstract}
Spectral sky radiance $(380-760 \mathrm{~nm})$ is derived from measurements with a hemispherical sky imager (HSI) system. The HSI consists of a commercial compact CCD (charge coupled device) camera equipped with a fish-eye lens and provides hemispherical sky images in three reference bands such as red, green and blue. To obtain the spectral sky radiance from these images, non-linear regression functions for various sky conditions have been derived. The camera-based spectral sky radiance was validated using spectral sky radiance measured with a CCD spectroradiometer. The spectral sky radiance for complete distribution over the hemisphere between both instruments deviates by less than $20 \%$ at $500 \mathrm{~nm}$ for all sky conditions and for zenith angles less than $80^{\circ}$. The reconstructed spectra of the wavelengths $380-760 \mathrm{~nm}$ between both instruments at various directions deviate by less than $20 \%$ for all sky conditions.
\end{abstract}

\section{Introduction}

The knowledge of spatial and spectral sky radiance distribution is important for many applications. For example, the sky radiance is used for studying the optical properties of mineral dust (Li et al., 2007) and bidirectional reflectance distributions (Deering and Eck, 1987). The optical thickness and the size distribution of aerosol were derived from the measured sky radiance using an inversion algorithm (Nakajima et al., 1996; Dubovik and King, 2000). The aerosol phase function and single scattering albedo can be determined from the sky radiance as well (Velmeulen et al., 2000). The spectral sky radiance is also used for the computation of the irradiance on surfaces, which is applied in design and performance investigations of spectral selective energy devices, such as photovoltaic (PV) systems (Steven and Unsworth, 1997; Hernández-András et al., 2001).

The variation of the sky radiance has been observed and analysed under different sky conditions by using several types of instruments (Dorno, 1911; Bener, 1963; Blumthaler et al., 1996; Ricchiazzi et al., 2000; Huber et al., 2004). Wuttke and Seckmeyer (2006) and Wuttke et al. (2006) performed spectral sky radiance measurements over the snowcovered surface in Antarctic environments in order to investigate the impact of high snow albedo on the sky radiance. A recent study by Pissulla et al. (2009) presented an intercomparison of spectral radiance measurements in the UV and visible wavelengths with high spectral resolution. These measurements were conducted by using five different instruments from three locations. The deviation of the measured spectral sky radiance varied between 3 and $35 \%$ depending on the wavelength, location and the instruments.

Most of these spectral radiance observations have been compared with the results from a radiative transfer model. These comparisons were mostly under cloud-free conditions and under partly cloudy conditions. However, the equipment for measuring spectral radiance distributions is relatively non-mobile and expensive, which leads to insufficient spectral radiance data. Therefore, some researchers have applied all-sky images to determine the spectral sky radiance, which has great benefits in terms of acquisition time, mobility and cost.

The development of all-sky imagery for ground-based sky radiance observations has taken over a century. Voss and Zibordi (1989) presented the sky radiance distribution performed by the electro-optics fisheye radiance distribution camera system (RADS) and validated with radiance data from a hand-held contrast reduction meter. The sky radiance 
distribution from the same system was also compared with sky radiance simulations (Zibordi and Voss, 1989).

The whole-sky imager was developed for ground-based cloud and radiance measurements (Shields et al., 1998; Feister et al., 2000). The grey level of a monochromatic CCD (charge coupled device) camera has been used to derive the sky radiance distribution for the different sky conditions (Huo and Lu, 2009) and the gray values combined with the radiometric calibration have been applied to obtain the spectral radiance (Rossini and Krenzinger, 2007). The spectral skylight from images using a linear pseudo-inverse has been presented (López-Álvarez et al., 2008). Recent research by Román et al. (2012) investigated the spectral sky radiance at three effective wavelengths from hemispherical sky images. A matrix calibration described the relationship between the output signal of images and reference values of the simulated sky radiance were proposed. The camera-based radiance has been estimated and validated with the sun photometer. However, validation of the spectral sky radiance distribution for the whole hemisphere with an accurate instrument has not been performed. Furthermore, the matrix calibration applied in this work is only valid when the spectral response of the CCD sensor is linear.

In contrast to Román et al. (2012), we propose a method to obtain the spectral sky radiance distribution from all-sky images for the complete spectrum in the visible part (380$760 \mathrm{~nm}$ ) using the non-linear regression technique. The instrumentation and the methods for retrieving the spectral radiance will be introduced in the next section, followed by spectral and spatial comparison of the sky radiance distributions derived by the hemispherical sky imager (HSI) system with the measured radiance distributions obtained by a CCD spectroradiometer.

\section{Instruments and data}

\subsection{The hemispherical sky imager (HSI) system}

The hemispherical sky imager (HSI) was designed and developed at the Institute of Meteorology and Climatology (IMuK), University of Hanover, Germany $\left(52.39^{\circ} \mathrm{N}\right.$, $9.70^{\circ} \mathrm{E}$, at $59 \mathrm{~m}$ a.s.1.). This system contains a Canon PowerShot G10 compact camera, equipped with a Dörr DHG fisheye lens, providing an image with a field of view (FOV) of about $183^{\circ}$ and encapsulated in a weather-proof housing for long-term outside operation. Hemispherical pictures can be taken and stored by the software every second. To keep the amount of storage low, an interval of $20 \mathrm{~s}$ is useful. More detailed descriptions of the HSI system are given in Tohsing et al. (2013). The camera has a CCD sensor with three channels such as red, green and blue. The spectral response of the camera channels has been investigated at IMuK laboratory by using a light source from the $1000 \mathrm{~W}$ lamp passed through a monochromator projected onto a reflectance plaque. At

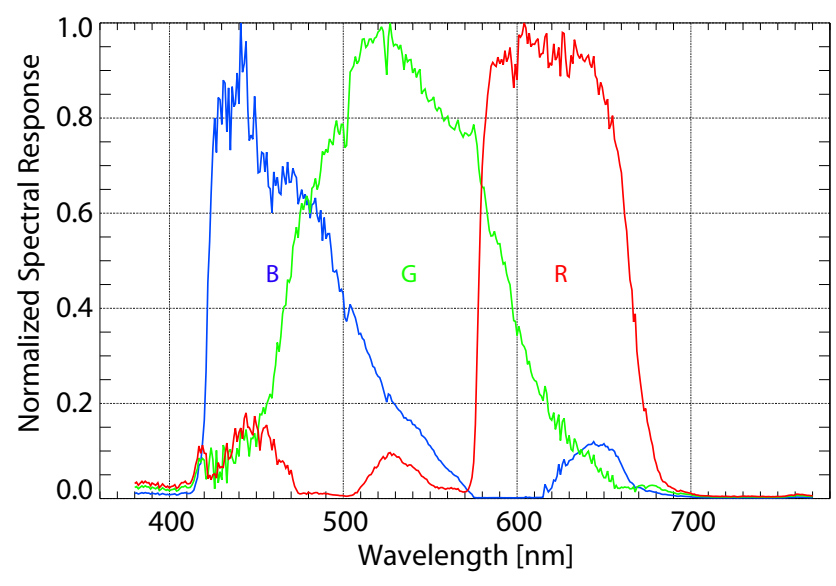

Figure 1. The relative spectral response of the Canon PowerShot G10 CCD sensor for the red, green and blue channel, investigated at the IMuK laboratory.

each wavelength images of the plaque were acquired and further analysed to obtain the sensor sensitivity. The resulting spectral response of the camera sensor used is shown in Fig. 1. The maximum responses of each channel are at the wavelengths of 602,527 and $441 \mathrm{~nm}$ for the red, green and blue channel respectively.

\subsection{The CCD Array spectroradiometer}

A CCD spectroradiometer system based on a CCD array spectrometer (S2000, Ocean Optics Inc., Dunedin, USA) and a positioning unit (SkyScanner, Czibula and Grundmann, Berlin, Germany) (Czibula and Grundmann, 2002) is used for measuring the spectral sky radiance distribution. Similar spectrometers have been used to measure solar and artificial radiation in Ansko et al. (2008); Kouremeti et al. (2008); Kreuter and Blumthaler (2009). The system is capable of measuring spectral sky radiance with a spectral resolution of $2 \mathrm{~nm}$ between 300 and $800 \mathrm{~nm}$ covering the visible part of the spectrum. The entrance optics has a $5^{\circ}$ FOV (Seckmeyer et al., 2010). The spectral sky radiance at a given zenith and azimuth angle is measured within five seconds. Based on earlier investigations with the main focus on the UV range, the estimated uncertainty of the spectral radiance measurements is between 5 and $10 \%$. The methodology for calibration of the CCD spectroradiometer, which is performed at the beginning of the measurement, is described in Pissulla et al. (2009) and has been applied to spectral radiance measurements in the visible range as explained in Seckmeyer et al. (2010). The effects of uncertainties on experimental integrals for the UV part have been described in Cordero et al. (2008) and further details on the extension to the visible range can be found in Wuttke and Seckmeyer (2006) and Wuttke et al. (2006). 
Table 1. Measuring description of the CCD spectroradiometer and the HSI system for spectral sky radiance reproduction.

\begin{tabular}{llll}
\hline Date & $\begin{array}{l}\text { Hour measuring } \\
\text { (UTC) }\end{array}$ & Sky conditions & Purpose \\
\hline 19 Oct 2012 & $05: 30-17: 27$ & Partly cloudy & Validation \\
20 Oct 2012 & $05: 30-16: 44$ & Partly cloudy & Validation \\
21 Oct 2012 & $10: 23-17: 21$ & Cloud-free & Validation \\
22 Oct 2012 & $07: 17-15: 23$ & Overcast & Validation \\
23 Oct 2012 & $06: 30-16: 21$ & Overcast & Training \\
26 Oct 2012 & $05: 30-17: 20$ & Partly cloudy & Validation \\
28 Oct 2012 & $12: 41-16: 00$ & Cloud-free & Training \\
31 Oct 2012 & $12: 25-16: 31$ & Partly cloudy & Validation \\
\hline
\end{tabular}

\subsection{Data}

Spectral sky radiance data were obtained by mounting the CCD spectroradiometer onto the Skyscanner platform, therefore allowing a variety of zenith and azimuth angles to be viewed, and comparing these results with the concurrent images from the HSI system. These data are categorized into two types, the first one to establish a training sample and the second data set to be used for the validation. Table 1 shows when the measurements and images are taken as well as the corresponding sky conditions.

The measurement of a radiance distribution for the whole hemisphere conducted with the CCD spectroradiometer has a scan pattern of 113 points and takes about $12 \mathrm{~min}$. Since HSI images are taken every $20 \mathrm{~s}$, a total of 35 images is within a complete scanning measurement of the CCD spectroradiometer. In order to use the radiance distribution of the CCD spectroradiometer for the validation, a synchronized HSI image is constructed. For this synchronized HSI image the pixels of each scan point were taken from the corresponding all-sky image, depending on the time of measurement by the CCD spectroradiometer. The corresponding sky patterns constructed from the HSI system under different sky conditions can be seen in Figs. 4d, 5d and 6d.

\section{Methods}

Sky radiance spectra from a limited number of discrete bands are derived from all-sky images. Due to the non-linear behaviour of the camera sensor (see Fig. 2) a non-linear regression approach has been applied to obtain all of the spectra in the visible region. This regression method has been applied in various spectral reconstruction applications as described in Johnsen et al. (2008), Milton and Rollin (2006) and Dahlback (1996). Non-linear regression is used to determine a relationship between two data sets - namely, the measured spectral radiance from the CCD spectroradiometer and the signal from the three channels of the HSI image. In the regression process, the optimum correlation was acquired with
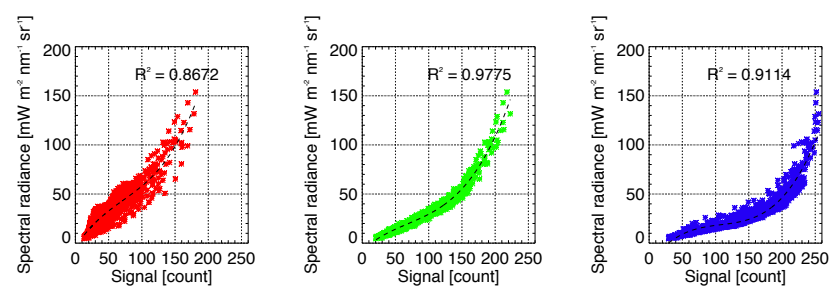

Figure 2. Non-linear relationship between the measured spectral sky radiance and the signal count for the three channels red, green and blue of the HSI system. The data are used as a training data set and were recorded on 28 October 2012 for the wavelength $500 \mathrm{~nm}$. The $R^{2}$ in the plots represent the correlation coefficients.

a variety of independent variables and the predictor variables were selected (Lütjohann, 1970).

The following two data sets were used in the training of the regression model for cloud-free condition. The first data set contains spectral sky radiance measurements, conducted with the CCD spectroradiometer in the wavelength range from 380 to $760 \mathrm{~nm}$ on 28 October 2012 from 12:00 to 16:00 UTC. The second data set consists of the signal counts of the three reference bands measured by the HSI system also on 28 October 2012, which have a maximum spectral response at the wavelength of $441 \mathrm{~nm}$ for the blue channel, $527 \mathrm{~nm}$ for the green channel and $602 \mathrm{~nm}$ for the red channel. In this case, the spectral direct radiance from the CCD spectroradiometer as well as the counts from the HSI system at the circumsolar area or about $20^{\circ}$ around the sun were excluded in the regression model.

At each wavelength (i.e. each regression equation), the measured spectral sky radiance was treated as a function of the signal of red or green or blue counts, which range from 0 to 255 counts. Therefore, there are three non-linear regression equations for each wavelength and 1143 regressions for the complete spectrum. For different camera settings such as exposure time or aperture and due to the non-linear relationship between those settings and the count values different regression equations must be computed. In this work, the regression was performed for an exposure time of $1 / 1000 \mathrm{~s}$, an ISO number (defined as sensitivity of a camera to incoming light) of 80 and an aperture of $f / 40$. The results of the nonlinear regression equations and the selection of those equations for each wavelength is presented in Sect. 4.1.

For the case of cloudy sky conditions, the regression was performed for the training data set on 23 October 2012 from 06:00 to 16:00 UTC measured by the CCD spectroradiometer and the HSI system. Except for the different training data set, the same method as described for the cloud-free condition was used. The reason for two training data sets (cloud-free and cloudy) is due to the high sensitivity of the HSI channel to cloud cover. The maximum values can reach 255 counts for bright clouds, whereas blue sky has at most about 120 counts. The use of one and the same training data set for 
both conditions would lead to great deviation in the spectral radiance reconstruction.

Under intermediate sky, the FOV of some scanning points is not completely filled with either clouds or blue sky, as shown in Fig. 6d. In these cases, it is quite difficult to decide whether the cloud-free or cloudy sky regression model is more suitable. Therefore, the ratio of the count values of the red and blue channel (called sky index) was investigated to separate blue sky and white clouds (Yamashita et al., 2004). The sky index is expressed as

Sky Index $=\frac{B-R}{B+R}$,

where $B$ is the count value of the blue channel and $R$ the count value of the red channel of the HSI system.

Eventually, all pixels of each scanning point were averaged to gain one representative sky index. The investigations showed that an average of sky index greater than 0.25 indicates blue sky. Thus, for these values, the cloud-free regression model will be applied for the spectral reconstruction, otherwise the sky element is covered by clouds.

A major challenge of this regression technique is that the collinearity between the predictor variables, which creates the regression relationship, is very specific to the training data set from which they are derived. Therefore, it would be unsuccessful to apply the same coefficients to other data set without a test of their suitability. Moreover, the training data set contains solar zenith angles $60-90^{\circ}$ due to the fact that the measurements have been performed in wintertime. For the validation of the spectra reproduction method, the spectral radiance was calculated for an independent image data set from the HSI system during one week of the campaign acquired under different sky conditions as shown in Table 1. A discrepancy between the spectral sky radiance from the HSI system and the CCD spectroradiometer is presented as a ratio and also in terms of mean bias difference (MBD) and root mean square difference (RMSD). MBD is determined (Iqbal, 1983) by

$\mathrm{MBD}=\frac{\frac{\sum_{i=1}^{N}\left(R_{\text {cam }}-R_{\text {mea }}\right)}{N}}{\overline{R_{\text {mea }}}} \times 100 \%$,

where $R_{\text {cam }}$ is the sky radiance derived from the HSI system, $R_{\text {mea }}$ is the measured sky radiance from the CCD spectroradiometer, $\overline{R_{\text {mea }}}$ is the averaged sky radiance obtained from the CCD spectroradiometer and $N$ is the number of measurement data. The RMSD measures the variation between the computed values and the measured values. RMSD is expressed as follows (Iqbal, 1983):

$\mathrm{RMSD}=\frac{\sqrt{\frac{\sum_{i=1}^{N}\left(R_{\mathrm{cam}}-R_{\mathrm{mea}}\right)^{2}}{N}}}{\overline{R_{\text {mea }}}} \times 100 \%$
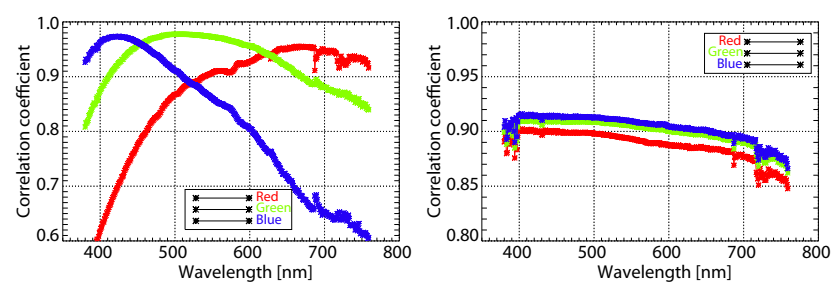

Figure 3. The optimum correlation coefficient $\left(R^{2}\right)$ derived from the comparison with the CCD spectroradiometer and the digital numbers of the three channels of the images from the HSI system for cloudless sky measured on 28 October 2012 (left) and on 23 October 2012 for cloudy sky (right).

The results of the comparison between camera-based and measured spectral sky radiance are presented in Sects. 4.2 and 4.3.

\section{Results}

\subsection{Non-linear regression model}

The synchronized data sets collected on 23 and 28 October 2012 by the CCD spectroradiometer and the HSI system were used as training data sets to determine the non-linear regression equations. Figure 2 shows the non-linear regression fitted by a third-degree polynomial and the correlation coefficient $\left(R^{2}\right)$ at the wavelength $500 \mathrm{~nm}$ for the three channels. The resulting plot in Fig. 3 presents the variation of the correlation coefficients of the training data for cloud-free sky on 28 October 2012 (left) and for cloudy sky on 23 October 2012 (right) plotted against the visible wavelength. For blue sky, the best correlation coefficients are at the centre of each band, which has a correlation coefficient of $R^{2}>0.95$ and the correlation coefficients of each channel vary with the wavelength that corresponds to the camera sensor response. The coefficients for cloud-free data of the red channel vary mostly in the region around $680-760 \mathrm{~nm}$ due to water absorption lines (Liou, 2002). In the case of cloudiness (see Fig. 3 right), there are variations of the correlation coefficients at wavelength less than $400 \mathrm{~nm}$ due to the absorption by ozone and in the $680-760 \mathrm{~nm}$ wavelength range, otherwise the correlation coefficients are quite constant ranging from about 0.85 to 0.92 .

Therefore, the general equations of the non-linear regression models for each wavelength $(\lambda)$ can be expressed by the following equations:

$$
\begin{aligned}
& L_{\mathrm{cam}, \mathrm{r}}=a_{0, \lambda}+a_{1, \lambda} R+a_{2, \lambda} R^{2}+a_{3, \lambda} R^{3} \\
& L_{\mathrm{cam}, \mathrm{g}}=b_{0, \lambda}+b_{1, \lambda} G+b_{2, \lambda} G^{2}+b_{3, \lambda} G^{3} \\
& L_{\mathrm{cam}, \mathrm{b}}=a_{0, \lambda}+c_{1, \lambda} B+c_{2, \lambda} B^{2}+c_{3, \lambda} B^{3}
\end{aligned}
$$

where $L_{\text {cam, r }}, L_{\text {cam, g }}, L_{\text {cam, b }}$ are the sky radiance values obtained from the red, green and blue channels of the images. 
$R, G$ and $B$ are the signal counts from the red, green and blue channel. $a, b$ and $c$ are the wavelength dependent coefficients of the regression equations.

The regression model for each wavelength has been selected by comparing the best correlation coefficients of the three channels. As presented in Fig. 3 (left), the blue channel yields the best correlation coefficients in the $380 \mathrm{~nm}$ to $450 \mathrm{~nm}$ wavelength range due to the sensitivity of this channel (see Fig. 1). The non-linear regression model from Eq. (6) has been applied to predict the spectral sky radiance in this region. The non-linear regression model from Eq. (5) dominated by the green channel is used to obtain the spectral sky radiance in the 451 to $620 \mathrm{~nm}$ wavelength range. Spectral sky radiance in the last part of the spectrum from 621 to $760 \mathrm{~nm}$ has been calculated by using the non-linear regression from the red channel as expressed in Eq. (4). Finally, the spectral sky radiance using the above three regression relationships may be estimated for the entire visible spectrum. The result of the application of these regression models to the independent validation data set of the synchronized HSI images is presented in Sects. 4.2 and 4.3.

\subsection{Comparison of spectral radiance distribution at $500 \mathrm{~nm}$ from CCD spectroradiometer and HSI system}

Examples of the spectral sky radiance at $500 \mathrm{~nm}$ for the whole hemisphere measured with the CCD spectroradiometer are compared with the spectral radiance from the HSI images for different sky conditions as shown in Figs. 4-6. Figure 4 shows the measurement and the comparison for cloud-free sky on 21 October 2012 at 13:12 UTC (scanning period is 13:12-13:24 UTC): (a) the measured spectral sky radiance from $\mathrm{CCD}$ spectroradiometer and interpolated for the whole sky (b) the spectral radiance distribution calculated from the HSI system, (c) the ratio between the camerabased and measured spectral sky radiation compared point by point (no interpolation) and (d) 113 points of the synchronized HSI image reconstructed from about 35 images depending on the scanning time. The size of the illustrated scan points in Fig. $4 \mathrm{~d}$ corresponds to the $5^{\circ} \mathrm{FOV}$ of the CCD spectroradiometer. Although the FOV of the scanning pattern is $5^{\circ}$ (Fig. 4d), the scanning points showing the ratio (Fig. 4c) are plotted with a FOV of $10^{\circ}$ for clarity of the presentation. A yellow star represents the position of the sun with its corresponding solar zenith angle (SZA). Due to the obstacles of buildings and trees, the spectral radiance for zenith angles higher than $80^{\circ}$ is not considered. Measurements in the circumsolar region are not included in the regression model, since both instruments suffer from oversaturation due to their limited dynamic range. Internal reflections of direct sunlight can cause ghost images in some parts of the HSI image and lead to an overestimation of the spectral sky radiance of about 2-5\% (Tohsing et al., 2013).
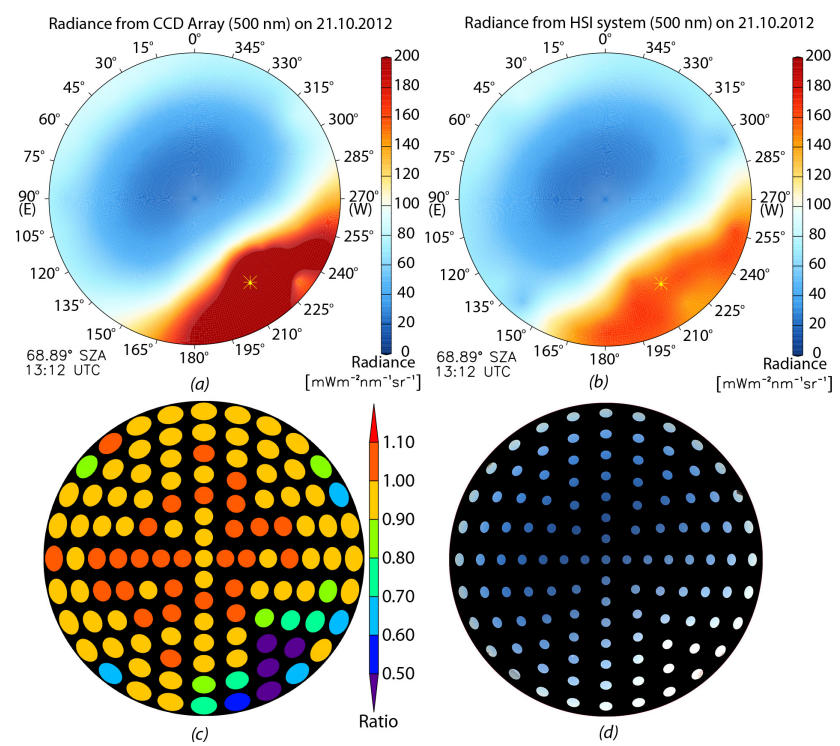

Figure 4. Spectral radiance distribution at $500 \mathrm{~nm}$ measured by (a) CCD spectroradiometer and (b) HSI system on 21 October 2012 at 13:12 UCT $\left(68.89^{\circ}\right.$ of solar zenith angle (SZA)) for cloud-free condition. (c) Ratio between the computed spectral radiance of the HSI system and the measured spectral radiance of the CCD spectroradiometer (HSI system/CCD spectroradiometer). Although the FOV of the scanning pattern is $5^{\circ}$ (see d), the scanning points showing the ratio are plotted with a FOV of $10^{\circ}$ for clarity of the presentation. The synchronized HSI image showing the $5^{\circ} \mathrm{FOV}$ pattern of the scanning points from the CCD spectroradiometer is illustrated in (d).

A rim at the horizon with higher values of spectral sky radiance can be observed, in contrast to the spectral sky radiance at the zenith. For the spectral sky radiance distribution, in general, the brightness of the horizon which is dominated by the Rayleigh scattering (Liou, 2002) increases with increasing wavelength and can clearly be observed in the measurements with the CCD spectroradiometer. From the results of the spectral sky radiance derived from the HSI system, the horizon brightening could not be identified at short wavelengths $(400 \mathrm{~nm})$ but could be distinctly observed at longer wavelengths $(600 \mathrm{~nm})$, which is not shown in the plots. The variation of the spectral sky radiance distribution at the wavelength $500 \mathrm{~nm}$ deviates by up to $10 \%$ for the whole hemisphere excluding the circumsolar region as shown in the ratio plot of Fig. 4c.

Figure 5 shows the comparison of the spectral sky radiance for the whole hemisphere for overcast sky conditions observed on 22 October 2012 at 09:29 UTC (scanning period, 09:29-09:41 UTC). The camera-based spectral radiance agrees well with the measured spectral sky radiance with a deviation up to $5 \%$ for zenith angles less than $80^{\circ}$. The result of this comparison can be seen in the ratio plot (Fig. 5c). The spectral radiance for zenith angles greater than $80^{\circ}$ were not considered due to obstructions at the horizon. 

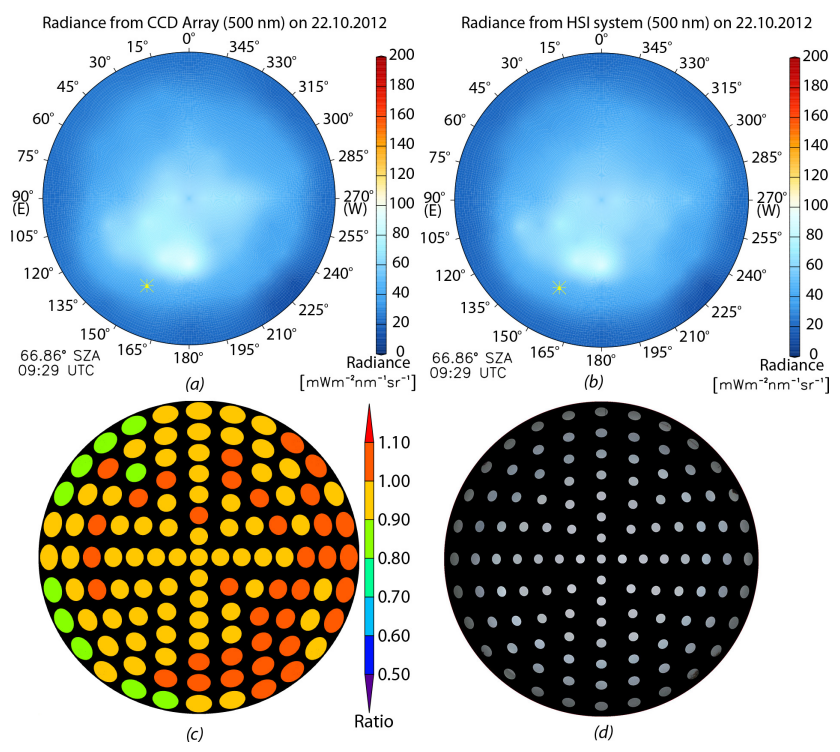

Figure 5. Spectral radiance measured at the wavelength $500 \mathrm{~nm}$ by the CCD spectroradiometer (a) and HSI system (b) on 22 October 2012 at 09:29 UCT $\left(66.86^{\circ}\right.$ of SZA) under overcast sky. (c) Ratio between the computed spectral radiance from the HSI system and the measured spectral radiance by the CCD spectroradiometer (HSI system/CCD spectroradiometer). Although the FOV of the scanning pattern used is $5^{\circ}$ (see d), the scanning points showing the ratio are plotted with a FOV of $10^{\circ}$ for clarity. The synchronized HSI image showing the $5^{\circ} \mathrm{FOV}$ pattern of the scanning points from the CCD spectroradiometer is illustrated in (d).

The validation for partly cloudy sky, measured on 26 October 2012 at 12:01 UTC (scanning period, 12:01-12:13 UTC), is presented in Fig. 6. Most of the sky elements with a zenith angle greater than $50^{\circ}$ were covered by clouds, while the zenithal area was cloudless. The sky index was determined and applied for separating blue sky and cloudy areas.

The comparison shows that the spectral radiance distribution of the blue sky at the zenith derived by the HSI system agrees well with the measurement with a deviation up to $10 \%$. The same deviation is observed at scanning points that are fully covered by clouds. If scanning points are covered by a small degree by clouds, the HSI system overestimates the spectral sky radiance. In this case the deviation is less than $20 \%$. However, the spectral sky radiance of bright clouds is overestimated by the HSI system.

The values of the MBD and RMSD of the compared sky radiance measurements for the wavelengths of 400, 500 and $600 \mathrm{~nm}$ during the measurement campaign under different sky conditions are summarized in Table 2 . The HSI system tends to underestimate the spectral sky radiance with respect to the CCD spectroradiometer as presented by the MBD. The MBD and the RMSD of the spectral sky radiance in the case of partly cloudy sky between both instruments were found to be higher than for completely cloud-free and overcast skies.
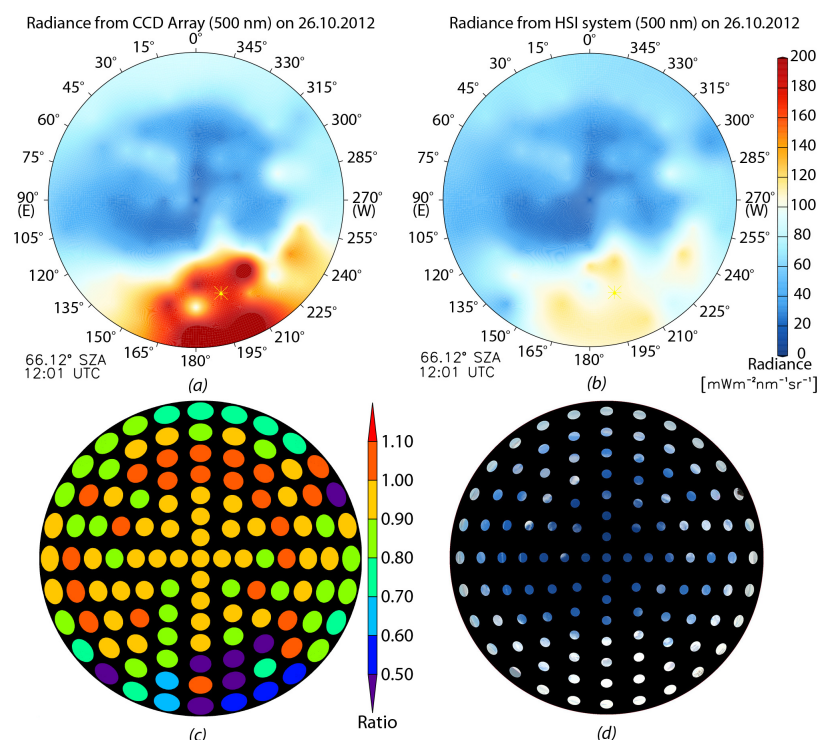

Figure 6. Spectral radiance measured at the wavelength $500 \mathrm{~nm}$ by the CCD spectroradiometer (a) and HSI system (b) on 26 October 2012 at 12:01 UCT $\left(66.12^{\circ}\right.$ of SZA) under intermediate sky. Plot (c) shows the ratio between the computed spectral radiance from the HSI system and the measured spectral radiance by the CCD spectroradiometer (HSI system/CCD spectroradiometer). Although the FOV of the scanning pattern used is $5^{\circ}$ (see d), the scanning points showing the ratio are plotted with a FOV of $10^{\circ}$ to achieve a better illustration. The synchronized HSI image showing the $5^{\circ}$ FOV pattern of the scanning points from the CCD spectroradiometer is illustrated in (d).

\subsection{Comparison of spectral radiance from CCD spectroradiometer and HSI system for the visible spectrum}

The spectral sky radiance obtained for the whole visible spectrum of the wavelength from 380 to $760 \mathrm{~nm}$ by the HSI system was compared with the measurements of the three different sky conditions mentioned in Sect. 4.2. For each sky condition, two points of the scan pattern were chosen to represent the spectral validation.

In Fig. 7 the reconstructed spectra from 21 October 2012 at 13:12 UTC for the scanning points with zenith angles of $36^{\circ}$ and $72^{\circ}$ and azimuth angles of $0^{\circ}$ and $105^{\circ}$ are illustrated. The validation shows that the camera-based spectral sky radiance agrees well with the spectral radiance from the CCD spectroradiometer and has a deviation of less than $10 \%$ in the 380 to $700 \mathrm{~nm}$ wavelength range as shown in the ratio plots of Fig. 7. For wavelengths greater than $700 \mathrm{~nm}$ the deviation can be up to $30 \%$ and could be caused by the absorption band of the water vapour (Liou, 2002) and the detection threshold of the CCD spectroradiometer in this wavelength region.

Figure 8 shows the radiance spectra for the overcast situation observed on 22 October 2012 at 09:29 UTC at the zenith and the scan point with the zenith angle of $48^{\circ}$ and the 


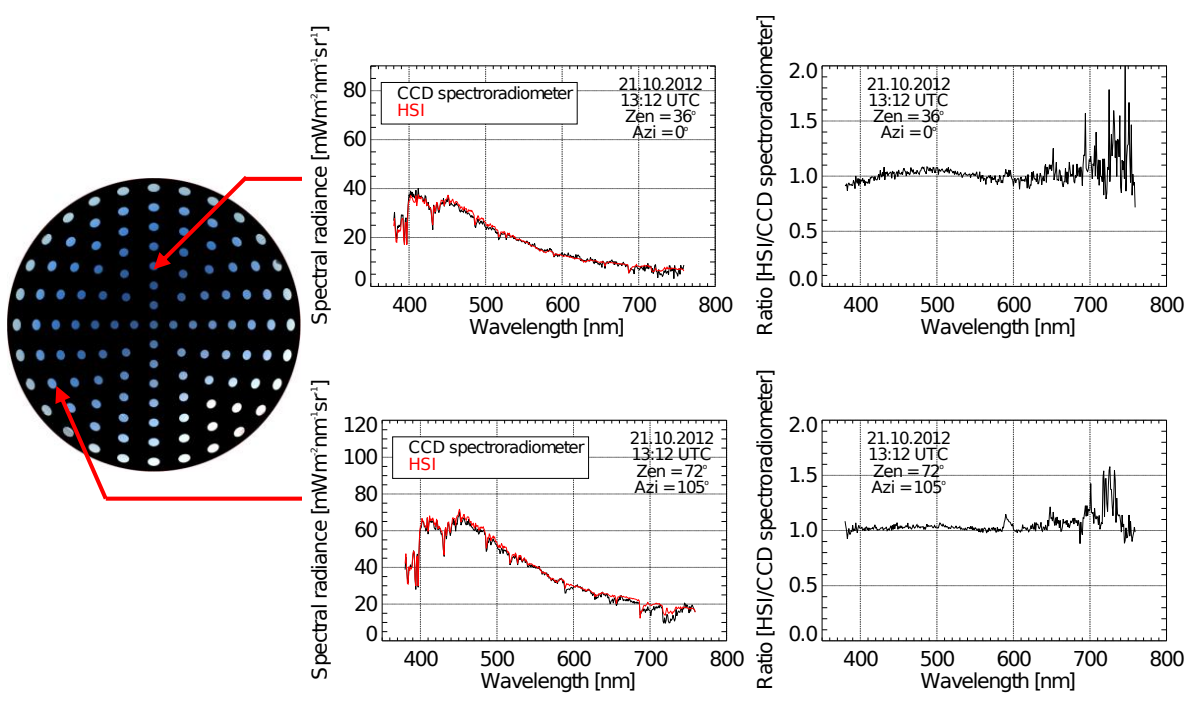

Figure 7. Spectral radiance measured on 21 October 2012 at 13:12 UTC by the CCD spectroradiometer and the HSI system for the scanning points with azimuth and zenith angles of $0^{\circ}$ and $36^{\circ}$ (upper panel) and $105^{\circ}$ and $72^{\circ}$ (lower panel). The ratio between the measured and computed spectral radiance is shown on the right.
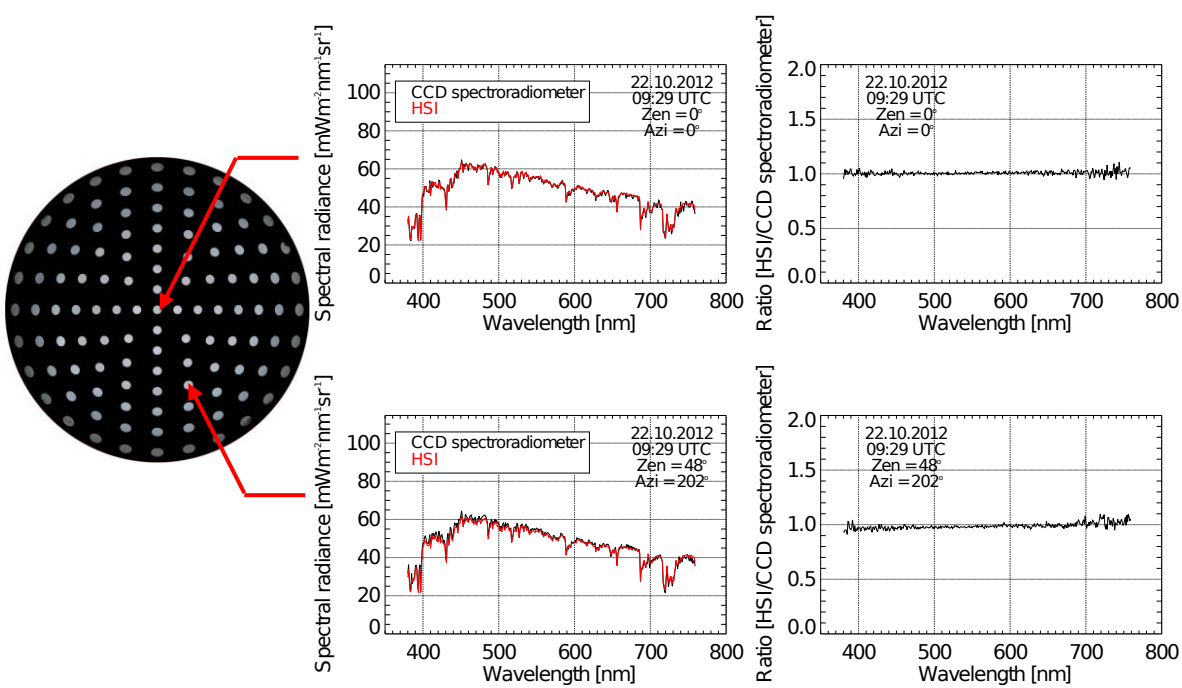

Figure 8. Spectral radiance on 22 October 2012 at 09:29 UTC measured by the CCD spectroradiometer and the HSI system at the zenith (upper panel) and for azimuth angle of $202^{\circ}$, zenith angle of $48^{\circ}$ (lower panel). The ratio between the measured and computed spectral radiance is shown on the right.

azimuth angle of $202^{\circ}$. The spectral radiance from the HSI image agrees very well with the measured spectral zenith radiance from the CCD spectroradiometer with a deviation of less than $10 \%$ in the visible spectrum.

As shown in Fig. 9, the radiance spectra were also determined by the HSI system for a broken cloud condition observed on 26 October 2012 at 12:01 UTC. The scanning point with the zenith angle of $48^{\circ}$ and the azimuth angle of $67^{\circ}$, shown in the upper panel, is covered up to one half with clouds. The sky index was found to be close to 0.22 . Therefore, the regression model for cloudy conditions was selected. The resulting value from the HSI system is slightly higher than from the CCD spectroradiometer but the deviation is still within $10 \%$. In the lower panel, the plot of the scanning point with the zenith angle of $60^{\circ}$ and the azimuth angle of $162^{\circ}$, which was fully covered by cumulus clouds, is shown. The values of spectral sky radiance determined by the HSI system were smaller than the measured spectral radiance, with a deviation of up to $20 \%$ for wavelengths less than $700 \mathrm{~nm}$. For wavelengths greater than $700 \mathrm{~nm}$ the deviation can increase up to $40 \%$ around the water absorption band. The deviation between the camera-based spectral radiance by 

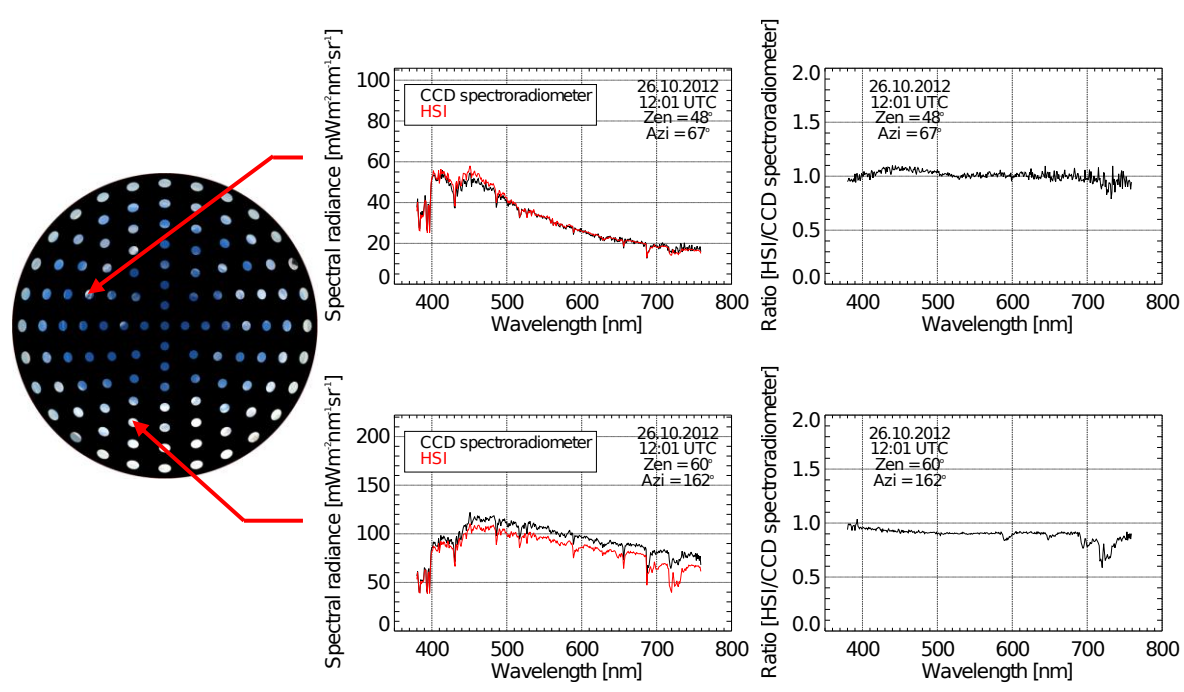

Figure 9. Spectral radiance measured on 26 October 2012 at 12:01 UTC by the CCD spectroradiometer and the HSI system for the scanning points with the azimuth and zenith angle of $67^{\circ}$ and $48^{\circ}$ (upper panel) and for the azimuth and zenith angle of $162^{\circ}$ and $60^{\circ}$ (lower panel). The ratio between the measured and computed spectral radiance is shown on the right. The agreement is considered to be satisfactory.

Table 2. The MBD and RMSD of the spectral sky radiance measured by the HSI system and the CCD spectroradiometer on various days corresponding to the sky conditions shown in Table 1.

\begin{tabular}{lrrrrrrrr}
\hline & \multicolumn{3}{c}{ MBD (\%) } & & \multicolumn{3}{c}{ RMSD (\%) } \\
\cline { 2 - 5 } \cline { 6 - 8 } Date & $400 \mathrm{~nm}$ & $500 \mathrm{~nm}$ & $600 \mathrm{~nm}$ & & $400 \mathrm{~nm}$ & $500 \mathrm{~nm}$ & $600 \mathrm{~nm}$ \\
\hline 19 Oct 2012 & -14.09 & -11.51 & -13.36 & & 19.13 & 16.11 & 17.12 \\
20 Oct 2012 & -17.76 & -15.22 & -18.60 & & 18.57 & 15.47 & 17.53 \\
21 Oct 2012 & -2.90 & -2.13 & -3.22 & & 10.46 & 6.20 & 12.14 \\
22 Oct 2012 & -3.09 & -2.12 & -2.46 & & 7.53 & 5.81 & 9.36 \\
26 Oct 2012 & -12.12 & -6.72 & -9.36 & & 13.41 & 14.34 & 15.71 \\
31 Oct 2012 & -10.41 & -7.40 & -10.08 & & 13.56 & 9.56 & 13.19 \\
\hline
\end{tabular}

the HSI system and the measured spectral radiance from the spectroradiometer for all directions and zenith angles (entire hemisphere excepting the sun position) was up to $20 \%$ for wavelengths less than $700 \mathrm{~nm}$. Sample results can be found in Sect. 4.2 for the wavelength of $500 \mathrm{~nm}$. For wavelengths greater than $700 \mathrm{~nm}$, the deviation can be up to $40 \%$ when some parts of scanning point were covered by clouds. Due to Mie scattering, the maximum radiance during cloudy situations is shifted from $400 \mathrm{~nm}$ for a cloud-free sky (Fig. 7, left upper panel) to $450 \mathrm{~nm}$ (Fig. 8, left upper panel) as described in Lenoble (1993). The shift of the radiance maximum to longer wavelengths was observed and investigated at different locations (Pissulla et al., 2009; Seckmeyer et al., 1994; Wuttke and Seckmeyer, 2006).

\section{Conclusions}

A non-linear regression model derived from the relationship between measurements and signal counts of the red, green and blue channels of hemispherical sky images was developed to derive the spectral sky radiance in the visible spectrum. The optimum non-linear regression equation for each wavelength was selected by using a training data set and the correlation coefficients between predicted radiance of the training set and the radiance from the measurements. For the validation, the camera-based spectral sky radiance from RGB image data obtained from the regression equations for different days under various sky conditions was calculated and compared with spectral sky radiance measurements by a CCD spectroradiometer.

The spectral sky radiance distribution for the whole hemisphere showed that the camera-based spectral sky radiance deviated from the sky radiance measured by the CCD spectroradiometer by less than $20 \%$ at $500 \mathrm{~nm}$ for all sky conditions and for zenith angle less than $80^{\circ}$. The comparison between the reconstructed visible spectra $(380-760 \mathrm{~nm})$ and the measured spectra with the CCD spectroradiometer also shows good agreement with a deviation of less than $20 \%$ for all sky conditions. From these investigations, it can be 
concluded that the HSI system can derive the spectral sky radiance distribution from hemispherical sky images with reasonable accuracy and reliability.

Acknowledgements. We acknowledge support by Deutsche Forschungsgemeinschaft and the Open Access Publishing Fund of Leibniz Universität Hannover.

Edited by: A. Macke

\section{References}

Ansko, I., Eerme, K., Lätt, S., Noorma, M., and Veismann, U.: Study of suitability of AvaSpec array spectrometer for solar UV field measurements, Atmos. Chem. Phys., 8, 3247-3253, doi:10.5194/acp-8-3247-2008, 2008.

Bener, P.: Der Einfluß der Bewölkung auf die Himmelsstrahlung, Theor. Appl. Climatol., 12, 442-457, doi:10.1007/BF02242986, 1963.

Blumthaler, M., Grübner, J., Huber, M., and Ambach, W.: Measuring spectral and spatial variations of UVA and UVB sky radiance, J. Geophys. Res., 23, 547-550, 1996.

Cordero, R. R., Seckmeyer, G., Pissulla, D., and Labbe, F.: Uncertainty of experimental integrals: application to the UV index calculation, Metrologia, 45, 1-10, 2008.

Czibula, G. and Grundmann, O.: Skyscanner - Gerätebeschreibung, Czibula and Grundmann Engeneering GbR, Berlin, 2002.

Dahlback, A.: Measurements of biologically effective UV doses, total ozone abundances, and cloud effects with multichannel, moderate bandwidth filter instruments, Appl. Optics, 35, 6514-6521, 1996.

Deering, D. W. and Eck, T. F.: Atmospheric optical depth effects on angular on anisotropy of plant canopy reflectant, Int. J. Remote Sens., 8, 893-916, 1987.

Dorno, C.: Studie über Licht und Luft des Hochgebirges, Vieweg und Sohn, Braunschweig, 1911.

Dubovik, O. and King, M. D.: A flexible inversion algorithm for retrieval of aerosol optical properties from Sun and sky radiance measurements, J. Geophys. Res.-Atmos., 105, 20673-20696, 2000.

Feister, U., Shields, J., Karr, M., Johnson, R., Dehne, K., and Woldt, M.: Ground-based cloud images and sky radiance in the visible and near infrared region from whole sky image measurement, in: EUMETSAT Satellite Application Facility Workshop, German Weather Service and World Meteorological Organization, 79-88, 2000.

Hernández-András, J., Romero, J., and Lee, J. R. L.: Colorimetric and spectro-radiometric characteristics of narrow-field-of-view clear skylight in Granada, Spain, J. Opt. Soc. Am., 18, 412-420, 2001.

Huber, M., Blumthaler, M., Schreder, J., Schallhart, B., and Lenoble, J.: Effect of inhomogeneous surface albedo on diffuse UV sky radiance at a high-altitude site, J. Geophys. Res., 109, 1-9, 2004.

Huo, J. and Lu, D.: Calibration and validation of an All-Sky Imager, Atmos. Ocean. Sc. Lett., 2, 220-223, 2009.

Iqbal, M.: An Introduction to Solar Radiation, Academic Press, Canada, 1983.
Johnsen, B., Kjeldstad, B., Aalerud, T. N., Nielsen, L. T., Schreder, J., Blumthaler, M., Bernhard, G., Topaloglou, C., Meinander, O., Bagheri, A., Slusser, J. R., and Davis, J.: Intercomparison and haromonization of UV index measurments from multiband filter radiometers, J. Geophys. Res., 113, 1-9, 2008.

Kouremeti, N., Bais, A., Kazadzis, S., Blumthaler, M., and Schmitt, R.: Charge-coupled device spectrograph for direct solar irradiance and sky radiance measurements, Appl. Optics, 47, 1594-1607, 2008.

Kreuter, A. and Blumthaler, M.: Stray light correction for solar measurements using array spectrometers, Rev. Sci. Instrum., 80, 096108, doi:10.1063/1.3233897, 2009.

Lenoble, J.: Atmospheric Radiative Transfer, Deepak Publishing, Hampton, 1993.

Li, Z., Goloub, P., Blarel, L., Damiri, B., Podvin, T., and Jankowiak, I.: Dust optical properties retrieved from groundbased polarimetric measurements, Appl. Optics, 46, 1548-1553, 2007.

Liou, K. N.: An Introduction to Atmospheric Radiation, Academic Press, Orlando, 2002.

López-Álvarez, M. A., Hernández-Andrés, J., Romero, J., Olmo, F. J., Cazorla, A., and Alados-Arboledas, L.: Using a trichromatic CCD camera for spectral skylight estimation, Appl. Optics, 47, H31-H38, 2008.

Lütjohann, H.: The stepwise regression algorithm seen from the statistician's point of view, Metrika, 15, 110-125, 1970.

Milton, E. J. and Rollin, E. M.: Estimating the irradiance spectrum from measurements in a limited number of spectral bands, Remote Sens. Environ., 100, 348-355, 2006.

Nakajima, T., Tonna, G., Rao, R., Boi, P., Kaufman, Y., and Holben, B.: Use of sky brightness measurements from ground for remote sensing of particulate dispersion, Appl. Optics, 35, 26722686, 1996.

Pissulla, D., Seckmeyer, G., Cordero, R. R., Blumthaler, M., Schallhart, Webb, A., Kift, R., Smedley, A., Bais, A. F., Kouremeti, N., Ced, A., Hermang, J., and Kowalewskig, M.: Comparison of atmospheric spectral radiance measurements from five independently calibrated systems, Photochem. Photobiol. S., 8, 516-527, 2009.

Ricchiazzi, P. J., Payton, A., and Gautier, C.: The Effect of Surface Albedo Heterogeneity on Sky Radiance, Tenth ARM Science Team Meeting Proceedings, San Antonio, Texas, 1-9, 2000.

Román, R., Antón, M., Cazorla, A., de Miguel, A., Olmo, F. J., Bilbao, J., and Alados-Arboledas, L.: Calibration of an all-sky camera for obtaining sky radiance at three wavelengths, Atmos. Meas. Tech., 5, 2013-2024, doi:10.5194/amt-5-2013-2012, 2012.

Rossini, E. G. and Krenzinger, A.: Maps of sky relative radiance and luminance distributions acquired with a monochromatic CCD camera, Sol. Energy, 81, 1323-1332, 2007.

Seckmeyer, G., Mayer, B., Erb, R., and Bernhard, G.: UV-B in Germany higher in 1993 than in 1992, Geophys. Res. Lett., 21, 577580, 1994.

Seckmeyer, G., Bais, A., Bernhard, G., Blumthaler, M., Drüke, S., Kiedron, P., Lantz, K., McKenzie, R. L., and Riechelmann, S.: Instruments to measure solar ultraviolet radiation - Part 4: Array spectroradiometers, Tech. rep., World Meteorological Organization, 2010. 
Shields, J. E., Johnson, R. W., Karr, M. E., and Wertz, J. L.: Automated day/night whole sky imagers for field assessment of cloud cover distributions and radiance distribution, in: Tenth Symposium on Meteorological Observations and Instrument, American Meteorological Society, 1998.

Steven, M. D. and Unsworth, M. H.: Standard distribution of clear sky radiance, Q. J. Roy. Meteorol. Soc., 103, 457-465, 1997.

Tohsing, K., Schrempf, M., Riechelmann, S., Schilke, H., and Seckmeyer, G.: Measuring high-resolution sky luminance distributions with a CCD camera, Appl. Optics, 52, 1564-1573, 2013.

Velmeulen, A., Deveax, C., and Herman, M.: Retrieval of the scattering and microphysical properties of aerosols from groundbased optical measurements including polarization. I. Method, Appl. Optics, 39, 6207-6220, 2000.

Voss, K. J. and Zibordi, G.: Radiometric and geometric calibration of a visible spectral electro-optic "fisheye" camera radiance distribution system, J. Atmos. Ocean. Tech., 6, 652-662, 1989.
Wuttke, S. and Seckmeyer, G.: Spectral radiance and sky luminance in Antarctica: a case study, Theor. Appl. Climatol., 85, 131-148, 2006.

Wuttke, S., Bernhard, G., Ehramjian, J., McKenzie, R., Johnston, P., ÓNeil, M., and Seckmeyer, G.: New spectroradiometers complying with NDSC standards, J. Atmos. Ocean. Tech., 23, 241-251, 2006.

Yamashita, M., Yoshimura, M., and Nakashizaka, T.: Cloud cover estimation using multitemporal hemispherical imageries, Int. Arch. Photogram. Rem. Sens. Spatial Inform. Sci., 35, 826-829, 2004.

Zibordi, G. and Voss, K. J.: Geometrical and spectral distribution of sky radiance: comparison between simulations and field measurements, Remote Sens. Environ., 27, 343-358, 1989. 